\title{
Nutrición, sobrepeso y obesidad en la edad infantil: Su relación con la actividad física deportiva
}

\author{
Nutrition, overweight and obesity in children's age: Their relations hip \\ with physical sports activity
}

Ángel Aníbal Sailema Torres. ${ }^{1}$ Jessica Paola Sailema Rios. ${ }^{2}$ Ángelo David Sailema Ríos. ${ }^{3} \&$ Marcelo Sailema Torres. ${ }^{4}$

\section{Abstract.}

Globally and also in Ecuador, overweight and childhood obesity have increased vertiginously in recent years; As a result of the above, obesity is considered by many experts as the epidemic of the century, entering the classification of chronic diseases. Given this global panorama that affects the quality of life and health of children and adults, the need to seek alternatives from early ages leading to prevention and control is imposed. Hence, the article presented has as a general objective: to reflect on the potential offered by the practice of physical sports activities from a multidisciplinary vision linked to proper nutrition for the prevention and control of overweight and obesity in children, especially if we take into account that it is precisely in this age range where adequate eating habits are formed that allow maintaining the necessary caloric levels and the motivation for the practice of progressively organized sports physical activities.

Based on a descriptive, non-experimental methodology with the use of theoretical methods such as historical -logical, synthetic and inductive -deductive analytical, documentary analysis and the debate on different theories around the subject treated, the main results are achieved: mean the importance of the practice of physical-sports activities and nutritional education in children as essential points

\footnotetext{
${ }^{1}$ Universidad Técnica de Ambato - Facultad de Ciencias Humanas y de la Educación Carrera de Cultura Física y Pedagogía de la Actividad Física y Deporte, aa.sailema@uta.edu.ec

${ }^{2}$ Universidad Técnica de Ambato - Facultad de Ciencias Humanas y de la Educación Carrera de Cultura Física y Pedagogía de la Actividad Física y Deporte, jessisailema@ hotmail.com

${ }^{3}$ Universidad Técnica de Ambato - Facultad de Ciencias Humanas y de la Educación Carrera de Cultura

Física y Pedagogía de la Actividad Física y Deporte, angelosailema@outlook.com, angelosailema@outlook.com

${ }^{4}$ Universidad de las Américas Quito - Facultad de Medicina. UDLA., ma.sailema@uta.edu.ec
} 
to prevent serious health problems caused by overweight or obesity from childhood, together the design of a program is provided.

Keywords: nutrition, overweight, obesity, children's age, sports physical activity.

\section{Resumen.}

A nivel mundial y también en Ecuador el sobrepeso y la obesidad infantil se han incrementado de manera vertiginosa en los últimos años; como consecuencia de lo anterior la obesidad es considerada por muchos expertos como la epidemia del siglo, entrando en la clasificación de enfermedades crónicas. Ante este panorama mundial que afecta la calidad de vida y la salud de la población infantil y adulta, se impone la necesidad de buscar desde edades tempranas alternativas conducentes a su prevención y control. De ahí que el artículo que se presenta tiene como objetivo general: reflexionar sobre las potencialidades que ofrece la práctica de actividades físicas deportivas desde una visión multidisciplinar unida a una nutrición correcta para la prevención y control del sobrepeso y la obesidad en la edad infantil, máxime si tenemos en cuenta que es precisamente en este rango de edad donde se forman los hábitos alimenticios adecuados que permiten mantener los niveles calóricos necesarios y la motivación para la práctica de actividades físicas deportivas organizada progresivamente. Basado en una metodología descriptiva, no experimental con el empleo de métodos teóricos como el histórico -lógico, el analítico sintético y el inductivo -deductivo, el análisis documental y el debate sobre diferentes teorías en torno al tema tratado, se logra como principales resultados: significar la importancia de la práctica de las actividades físicas- deportivas y la educación nutricional en la edad infantil como puntos esenciales para prevenir los serios problemas de salud que ocasiona presentar sobrepeso u obesidad desde la edad infantil, conjuntamente se aporta el diseño de un programa.

Palabras claves: nutrición, sobrepeso, obesidad, edad infantil, actividad física deportiva.

\section{Introducción.}

La situación respecto al sobrepeso y obesidad infantil y su relación con la actividad física en Ecuador, se refleja en las estadísticas que figuran en el Tomo I de la Encuesta Nacional de Salud y Nutrición (Ensanut, 2012).

Los resultados de la encuesta muestran un problema tanto por déficit como por exceso, pero lo más alarmante ha sido el aumento del sobrepeso y obesidad, el cual prácticamente se ha incrementado en un $200 \%$ en 30 años. Seis de cada 10 personas en Ecuador padecen sobrepeso u obesidad. 
La Ensanut también reveló que el sobrepeso/obesidad afecta en un 30\% a la población escolar (5-11 años), en un 26\% a los adolescentes (12-19 años) y en un 63\% a la población adulta de entre 19 y 60 años.

El estudio reflejó además que entre las múltiples causas que provocan este problema en Ecuador, tenemos al Sedentarismo y la baja práctica de Actividad Física.

En relación con el Sedentarismo, que está relacionado con la prevalencia de tiempo dedicado a ver televisión y videojuegos, los niños entre 5 y menores de 10 años, lo utilizan un $21,1 \%$ del tiempo del día, lo cual representa un tiempo mayor a 2 horas equivalentes a un $(17,4 \%)$ y a 4 horas $(3,7 \%)$ en otros casos, mientras que los adolescentes utilizan el $25,7 \%$ del tiempo del día, ocupando un tiempo mayor a 2 horas para un $(20,3 \%)$ y a 4 horas $(5,4 \%)$ respectivamente.

En relación a la Actividad Física se describe que la prevalencia de práctica de actividad física global en las personas se manifiesta de la siguiente forma: en el caso de adultos de 18 a 60 años, resultó un 12,1\% de Personas Inactivas del sexo masculino y del sexo femenino un 17,1\%. En el caso de las personas que realizan una Baja Actividad Física, resultaron 23,0 \% en el masculino y 36,7\% en el femenino, mientras que las personas que realizan una Mediana o Alta Actividad Física, un 64,9 \% son del sexo masculino y 46,2\% del sexo femenino, datos que fundamentan los resultados obtenidos.

El problema de la obesidad radica en que las personas con sobrepeso tienen el riesgo de desarrollar enfermedades crónicas no transmisibles, como diabetes mellitus tipo 2, hipertensión, enfermedades cardiovasculares y otros tipos de cáncer. De acuerdo a las cifras del Instituto Nacional de Estadísticas y Censos del Ecuador (INEC), estas precisamente constituyen algunas de las principales causas de muerte en Ecuador.

En los últimos dos años con el objetivo de frenar la obesidad y sobrepeso, se han establecido tres políticas públicas en Ecuador dentro de estas se encuentran: Etiquetado de alimentos, Regulación en el Sistema de Alimentación Escolar y la Ley para el Equilibrio de las Finanzas Públicas: aumento del impuesto para las bebidas alcohólicas y azucaradas, cigarrillos, entre otros. Las bebidas que se incluyeron en la Ley son las gaseosas y energizantes y otras bebidas azucaradas con un contenido mayor a 25 gramos de azúcar por litro (USD 0,17 por litro).

No obstante, a ser estas políticas públicas pensadas en bien de la sociedad, ellas son insuficiente para lograr eliminar o disminuir significativamente este flagelo, para ello, se necesita ampliar el horizonte y relacionarlo además con los cambios de hábitos y la concientización de la necesidad de realización de ejercicios físicos y precisamente inculcarse desde las edades tempranas. 
Ante todo, lo anterior cabe preguntarnos ¿Cómo contribuir a fomentar hábitos adecuados de alimentación unidos a la práctica sistemática de actividades Físicas deportivas conducentes a la prevención y control del sobrepeso y la obesidad en la edad infantil?

Es por esta razón que el objetivo general de este trabajo se centra en reflexionar sobre las potencialidades que ofrece la práctica de actividades físicas deportivas desde una visión multidisciplinar unida a una nutrición correcta para la prevención y control del sobrepeso y la obesidad en la edad infantil.

\section{Desarrollo.}

\section{El Sobrepeso u Obesidad- Realización de Actividad Física o Ejercicio Físico}

Al respecto debemos señalar que se considera a la obesidad como una enfermedad crónica y para muchos constituye la epidemia del actual siglo. Está caracterizada por un exceso de grasa en el organismo, traduciéndose en un aumento de peso. La misma se identifica a través del Índice de Masa Corporal (IMC= Peso $(\mathrm{kg}) / \mathrm{Talla} 2(\mathrm{~m})$ de un individuo), que según la Organización Mundial de la Salud (OMS), proporciona la medida más útil de sobrepeso y obesidad, determinando el primero con un IMC mayor o igual a $25 \mathrm{y}$ al segundo con uno mayor o igual que 30 , respectivamente, conforme a la tabla siguiente.

Tabla 1: Clasificación de la Obesidad [OMS]

\begin{tabular}{cc}
\hline INDICE DE MASA CORPORAL & CLASIFICACION DE LA OBESIDAD \\
\hline$<18,5$ & Insuficiencia Ponderal \\
$18,5-24,9$ & Peso normal \\
$\geq 25,0$ & Sobrepeso \\
$25,0-29,9$ & Preobosidad \\
$\geq 30,0$ & Obesidad \\
$30,0-34,9$ & Obesidad grado I \\
$35,0-39,9$ & Obesidad grado II \\
$\geq 40,0$ & Obesidad grado III \\
\hline
\end{tabular}

Fuente: Elaboración propia

No obstante, en relación a ello, coincidimos con (Roldán, Muñoz, \& Ochoa, 2010), en que debe prestarse atención al uso aislado del IMC (Indice de Masa Corporal), dado que puede llevar a resultados deficientes, debido a que específicamente, no discrimina a qué se debe el incremento del peso corporal, sea por la masa magra o por la masa grasa; así coincidimos con el criterio de (Roldán \& Rendón, 2013), quienes recomiendan se incorporen otras medidas antropométricas como los diferentes pliegos cutáneos, de esta manera se podrá evaluar de forma más precisa la proporción del tejido graso corporal y tener una mayor seguridad de que el exceso de peso, se corresponde realmente al tejido graso corporal y no por masa muscular. 


\section{La OMS y los patrones de crecimiento infantil- sobrepeso y obesidad.}

En el Boletín de la Organización Mundial de la Salud (OMS) de 2007, se recogen los resultados de la investigación "Elaboración de un patrón OMS de crecimiento de escolares y adolescentes" realizada por (M. De Onis, Onyango, Borghi, Siyam, Nishida, \& Siekmann); el cual tuvo como objetivo, elaborar curvas de crecimiento para escolares y adolescentes que concuerden con los Patrones de Crecimiento Infantil de la OMS para preescolares y los valores de corte del índice de masa corporal (IMC) para adultos.

Como uno de los resultados de la investigación realizada, resultó que el peso para la edad, no basta para seguir el crecimiento después de la infancia debido a su incapacidad para distinguir entre talla relativa y masa corporal; por esa razón la investigación ofrece el IMC para la edad, con el fin de complementar la talla para la edad en la evaluación de la delgadez (IMC bajo para la edad), el sobrepeso y la obesidad (IMC alto para la edad) y el retraso del crecimiento (talla baja para la edad) en los escolares y los adolescentes.

Las tablas de IMC para los niños y niñas de 5 a 19 años elaboradas recogen, la información necesaria de referencia para determinar el rango respectivo. O sea, en el caso del sobrepeso y la obesidad en niños y adolescentes, el crecimiento obliga a tener en cuenta la edad y el sexo, el cual, en función de la edad se comporta de la siguiente forma:

\section{Niños menores de 5 años}

En el caso de los niños menores de 5 años:

- El sobrepeso es el peso para la estatura con más de dos desviaciones típicas por encima de la mediana establecida en los patrones de crecimiento infantil de la OMS.

- La obesidad es el peso para la estatura con más de tres desviaciones típicas por encima de la mediana establecida en los patrones de crecimiento infantil de la OMS.

\section{Niños de 5 a 19 años}

En el caso de los niños de 5 a 18 años, el sobrepeso y la obesidad se definen de la siguiente manera:

- El sobrepeso es el IMC para la edad con más de una desviación típica por encima de la mediana establecida en los patrones de crecimiento infantil de la OMS.

- La obesidad es mayor que dos desviaciones típicas por encima de la mediana establecida en los patrones de crecimiento infantil de la OMS. 
En relación a ello y conforme a los anteriores autores y a (Barlow, 2007), se precisan percentiles y curvas de referencia, dependiendo de los grupos étnicos y nacionales representados en cada caso, de manera que entre los percentiles 85 y 95 se considera «sobrepeso», un percentil 95 o mayor se tipifica como «obesidad», y por encima del percentil 99 se clasifica como «obesidad severa».

En correspondencia a lo señalado, se hace significativo la clasificación que se realiza al respecto, para poder en base a ello, realizar un correcto direccionamiento de la actividad o del tipo de ejercicio físico a realizar. De acuerdo a la distribución de la grasa en el cuerpo (Barbany, 2002), clasifican la obesidad en Generalizada: cuando su distribución es pareja en todo el cuerpo sin predominio de ningún segmento, Androide: cuando su distribución es central, Visceral o Troncular y Ginecoide: cuando la grasa se distribuye predominantemente en el segmento inferior del cuerpo.

Ello trae a relucir la problemática sobre el efecto que realmente pueda aplicarse en disminuir la obesidad, ya sea la actividad física o el ejercicio físico, resultando necesario, ahondar en el alcance de estos conceptos.

Al respecto (Roldán \& Rendón, 2013), expresan que "la actividad física se puede definir como una acción que involucra gran parte de la masa muscular y produce una consiguiente elevación en el metabolismo energético. Por lo tanto, la actividad física involucra desde actividades de muy poca intensidad como caminar lentamente o estar sentado trabajando en el computador, hasta actividades intensas a nivel deportivo. Las primeras no permiten generar cambios o adaptaciones celulares, de órganos o sistemas corporales".

Conforme a lo expresado, la actividad física como tal, representa una actividad que genera un gasto de energía; pero si esta actividad no se realiza metódicamente dosificada y organizada, en relación al propósito planteado, no generará los resultados esperados.

Ello implica que no debe recomendarse a una persona obesa la realización de alguna actividad física sin ninguna orientación, dado que la actividad puede ser insuficiente para el logro de disminuir peso corporal o por el contrario pudiera exagerar en la intensidad de la misma, produciendo lesiones osteomusculares, conforme se expresa en la Revista Politécnica ISSN 1900-2351, Año 9, Número 16, 201378.

\section{La realización del ejercicio físico como instrumento de disminución del peso corporal.}

En relación al ejercicio físico, (Sánchez, Pinilla, 1992) \& (Nelson, Rejeski, Blair, Duncan, Judge, King, Castañeda- Sceppa, 2007) plantean, que "es aquella actividad que se programa, se controla en intensidad y duración y frecuencia semanal, además de tener un objetivo claro para lograr efectos positivos para la salud". 
En esta dirección, la Intensidad constituye uno de los criterios de carga para dosificar los ejercicios, y el factor Capacidad Aeróbica Máxima (VO2) o la frecuencia Cardiaca de Reserva (HRR-por sus siglas en inglés), constituyen el elemento de control de la Intensidad del mismo.

En este ámbito; (Pollock \&Willmore, 1990), han distinguido que realizar ejercicios a una intensidad leve, representa cerca del $35 \%$ de la capacidad aeróbica máxima (VO2 máximo), realizar un ejercicio moderado se sitúa en un rango de intensidad entre el $50 \%$ y $75 \%$ del VO2 máximo y el ejercicio de alta intensidad está sobre el 75\% del VO2 máximo.

Coincidimos con (Barbany , 2002), en que son diversas las adaptaciones metabólicas que se producen como resultado del ejercicio y que, de hecho, son beneficiosas para el tratamiento de la obesidad, tales como el aumento del potencial oxidativo de las grasas, o sea, que resulte posible metabolizar grasas e hidratos de carbono de una forma aeróbica, lo que origina adaptaciones periféricas, como el aumento de la sensibilidad a la insulina, la disminución de los lípidos sanguíneos y el aumento del gasto cardiaco.

En conexión con diversos actores, (Wilmore, Després, Stanforth, Mandel, Rice, Gagnon, Bouchard, 1999)", (Aguilar,2007), (Janiszewski, \& Ross, 2009), con los cuales compartimos sus criterios, al plantear que "La realización de actividad física de forma regular cuenta con varias ventajas: tales como evitar la disminución de la tasa metabólica basal inducida por la dieta, disminuye todos los factores de riesgo asociados como Hipertensión Arterial, Diabetes Mellitus protege la masa magra, disminuye la ansiedad, la depresión y Mejora la composición corporal.

Además, y conforme a (Manson, Greenland, LaCroix, Stefanik, Mouton, Oberman, 2002), "la ejecución del ejercicio mejora el perfil lipídico, la composición corporal, la función cardiorrespiratoria, entre otros. Muchos de estos son independientes de la pérdida de peso; el riesgo cardiovascular disminuye tanto en el ejercicio intenso como en el leve o moderado".

Es por ello, que las metas en los ejercicios en la obesidad y de acuerdo a (Wong \& Murillo, 2004) tienen que estar muy definidas; si no hay comorbilidad, la meta a seguir es la pérdida de peso, pero si existe comorbilidad, hay que definir las metas apropiadamente: $\mathrm{Hb}$, glicosilada, valor de LDL y presión arterial”.

Según (Aguilar, Cordero, Ortegón Piñero, N. Mur Villar, Sánchez, Verazaluce, \& Sánchez López,2014), se ha podido constatar la eficacia del ejercicio físico en la reducción del sobrepeso y la obesidad de niños y adolescentes, así como que, el programa de actividad física más efectivo es el que combina ejercicios aeróbicos y anaeróbicos. 


\section{Nutrición. Restricción calórica.}

La nutrición debe realizarse de manera diferenciada para cada persona que intervenga en el programa de ejercicios, en las que el Licenciado en Cultura Física conozca las costumbres alimentarias de la persona, su forma de vivir, su historial clínico así como las complicaciones de salud que están añadidas a su obesidad. Ello exige conocer el historial dietético, de manera que se pueda elaborar una dieta tan personalizada como sea posible, adecuándola a los gustos, horarios, nivel económico y cultural y teniendo en cuenta las posibles enfermedades colaterales como DM, HTA, entre otros aspectos. Se debe realizar un control periódico, que permita darle seguimiento, para evaluar el comportamiento y así lograr la obtención de un óptimo resultado.

El conocimiento de los aspectos anteriores permitirá que se pueda planificar, en función del tipo y la intensidad de los ejercicios que se realizarán, una dieta balanceada, hipocalórica, que debe disminuir la energía suministrada, pero no la proporción de nutrientes necesarios para mantener un óptimo estado de salud.

Diversos autores, entre los que podemos citar a (Foz, Formiguera,1998) \& Medscape (2002), basados en estudios realizados, aconsejan disminuir un 35-40 \% de la energía de alimentación habitual. De igual manera expresan que mantener una dieta equilibrada consiste en un porcentaje de 50-60\% del total de la energía en carbohidratos y un $15 \%$ de proteínas, un 30-35\% de grasas, vitaminas y minerales recomendadas, presencia de fibras en la dieta y una ingesta adecuada de agua (1,5 litros al día).

Para (Fox, E. 1984) un tiempo razonable para la pérdida de peso es alcanzar la reducción del $10 \%$ del peso corporal en 6 meses de terapia. Se requiere de un balance energético negativo de $3500 \mathrm{kcal}$ para disminuir $1 \mathrm{lb}$ de grasa.

En el caso de pacientes con sobrepeso la disminución de 300 a $500 \mathrm{Kcal} /$ día resulta en la pérdida de peso de 0,5 a 1 libra/semana y un $10 \%$ de pérdida de peso en 6 meses.

Cuando predomina la obesidad, el déficit de 500 a $1000 \mathrm{kcal} /$ día que conduce a la pérdida de 1 a 2 libras/semana, y a un $10 \%$ de peso perdido en 6 meses.

De manera general, mayores restricciones son difíciles de cumplir por las personas con sobrepeso o con obesidad, dado que las pérdidas rápidas de peso originan o un alto índice de fracaso o la aparición de otras complicaciones para la salud.

Es por ello primordial planificar de antemano los tipos de ejercicios y las cargas de ejecución de los mismos, de manera diferenciada, en función de cada paciente. En conformidad a lo expresado por (Cevallos \& Castillo, 2018) "es muy importante tener en cuenta el tipo de actividad física que se prevé realizar, ya que, en función de las características de la misma, las capacidades implicadas en ella serán diferentes y, en consecuencia, los requerimientos nutricionales también lo serán. Como ejemplo de ello, 
la demanda de la cantidad de proteínas será mayor en las actividades de fuerza que en las de resistencia”.

En función de esto, se hace necesario de igual manera, como punto de partida, conocer las necesidades proteicas de una persona, conforme a ello, coincidimos con (Butterfield \& Calloway 1984), que indican que las necesidades proteicas diarias de una persona se encuentran en un rango que oscilan entre los 0,8 y los $1,2 \mathrm{~g} / \mathrm{kg} / \mathrm{d}$ siendo estas cantidades, suficientes para personas que realizan una actividad física aeróbica de baja intensidad, como ejemplo caminar.

En concordancia con lo explicado la incidencia de la nutrición de conjunto con un programa de ejercicios físicos para la disminución del peso corporal es fundamental, ya que lo adecuado para perder peso es disminuir la ingestión de calorías, pero ello debe realizarse en función de cada persona. Existe un consenso entre los especialistas que la dieta juega un papel primordial en el tratamiento de la obesidad y su incidencia en la pérdida de peso, pero siempre aparejado a un programa de ejercicios conlleva a que se obtengan los mejores resultados, para los niños, niñas y adolescentes con sobrepeso y obesos.

Esto nos lleva a pensar en la necesidad de fomentar hábitos adecuados de alimentación y práctica sistemática de actividad física deportiva desde edades tempranas, lo cual conlleva a una prevención oportuna de los niveles de sobrepeso y obesidad.

\section{Premisas para el Programa de actividad física para niños, niñas y adolescentes con} sobrepeso u obesos.

Los principales ejercicios que conforman los programas de actividad física, de forma general se agrupan en tres tipos: Ejercicios aeróbicos, anaeróbicos y de flexibilidad, no obstante, los que más se utilizan para combatir la obesidad son los aeróbicos y anaeróbicos.

Existe un criterio generalizado entre los especialistas, en que una vez que se inicie un programa de ejercicios, una tasa de pérdida de peso adecuada es de 0,5 kg/semana. El ejercicio propiamente facilita la pérdida de peso a largo plazo, por lo que se hace más difícil perder peso y mantenerlo que perder peso rápido, pues en este caso, la ganancia del peso también ocurre de una forma rápida. Debe tenerse en cuenta que el éxito para el paciente es mantener el peso perdido y de hecho también lo constituye para el especialista o licenciado de Educación Física.

Según (Micheli, 2001), "La utilización de las grasas como combustible principal del cuerpo durante el ejercicio, se da a partir de los 30 minutos de realizar ejercicio aeróbico de leve a moderada intensidad. No obstante, la pérdida de grasa máxima es en promedio de 4 a 6 Kg utilizando solo el ejercicio, y posteriormente no se perderá más”. De ahí que para que haya una significativa y constante reducción del peso asociado al ejercicio, se 
hace necesario que, durante la realización del programa de actividad física, exista una restricción de los ingresos calóricos de la persona, o sea, una dieta nutricional.

El aumento del porcentaje corporal de grasa y el déficit de tejido muscular son características que poseen las personas con obesidad. Es debido a ello, que el programa de un régimen de ejercicios, no debe realizarse sin una introducción previa, con los ejercicios adecuados, los que deben realizarse de manera escalonada y dosificada, para evitar lesiones.

En vistas a ello, lo primero que hay que hacer es comenzar los ejercicios, con el objetivo de producir cambios beneficiosos en la composición corporal, tales como, el aumento del tejido muscular, la disminución del tejido adiposo y la mejora del depósito de calcio en los huesos, así como en el nivel funcional de la persona, asociado al disfrute del ejercicio. Para lograr estos resultados, un tipo de ejercicio factible es el caminar diariamente 30 minutos. Ya una vez, vencida esta etapa, se podrá valorar la prescripción de un programa de ejercicios que incluya aspectos importantes, tales como: los tipos de actividad a realizar, las cargas específicas de trabajo, la duración de las actividades o sesiones a realizar (un mínimo de 30 minutos de 5 a 6 días en la semana), la intensidad de los ejercicios así como las acciones que deben preverse asociadas a problemas que pudieran originarse, ya sea ortopédicos u otros.

\section{Metodología.}

Se utilizó una metodología descriptiva, no experimental con el empleo de métodos teóricos como el histórico -lógico, el analítico sintético y el inductivo -deductivo, el sistémico-estructural funcional, acompañado del análisis documental y el debate sobre diferentes teorías en torno al tema tratado.

\section{Propuesta de soluciones.}

Una vez reflexionado en torno al tema de investigación, las teorías antecedentes y los aportes de diferentes autores en relación a la importancia de la atención multidisciplinar y preventiva del sobrepeso y la obesidad en la edad infantil, se asume la necesidad de realizar un conjunto de diferentes orientaciones para llegar a la concepción del Programa de Ejercicios Físicos previsto para niños con sobrepeso u obesidad.

Orientaciones para la concepción de un Programa de ejercicios físicos para niños con sobrepesos y obesos, mediante una adecuada praxis pedagógica.

- Incluir dentro de sus instrumentos de medición y control de los niños y niñas con sobrepeso y obesos, las tablas actualizadas de Índices de Masa Corporal (IMC) de niñas, niños y adolescentes entre 5 y 19 años, asumidas por la OMS. 
- Incorporar los conocimientos necesarios que permitan conocer la vinculación que tienen como elementos interactivos, el nivel de actividad física y la nutrición, en los niños con sobrepeso y obesos, en base a las especificidades propias de cada individuo.

- Realizar la planificación y selección de las diferentes actividades físicas en correspondencia con el tipo y características de cada niño o niña con sobrepeso u obeso, teniendo en cuenta sus reservas nutricionales y la dieta a realizar.

- Procurar la continuación de las actividades físicas planificadas siempre y cuando esté precedido de una adecuada alimentación, de manera que las mismas conlleven a la disminución paulatina del peso sin producir afectaciones a la salud.

- Trabajar en equipos multidisciplinares, enfocando los procesos de intervención en función de los objetivos de reducción del peso corporal de los niños y niñas incluidas dentro del programa.

- Valorar la realización de la actividad física desde una perspectiva de participación consciente de cada niño, niña o adolescente, en la cual se comprenda la relación entre la ingesta de nutrientes necesarios en función de las actividades físicas a realizar.

- Realizar un continuo control del comportamiento del programa, de manera diferencial por cada niño o niña, de manera que permita realizar las modificaciones necesarias para lograr los objetivos propuestos para cada etapa.

En relación a lo tratado, en el cuadro que se presenta a continuación, se expresa una concepción resumida, del Programa respecto a la incidencia de la nutrición en el sobrepeso y la obesidad en la edad infantil y adolescente así como la importancia primordial que presenta la realización de actividades y ejercicios físicos vinculados a la necesaria praxis del especialista de Educación Física, en las diferentes etapas de la realización de estas actividades, permitiendo la continuidad de la misma y la progresiva disminución del peso corporal de los niñas y niñas que realizan esta actividad, de manera saludable y sin daños a sus organismos. 
Figura 1: Esquema Concepción del Programa de actividad física para niños, niñas y adolescentes con sobrepeso u obesidad.

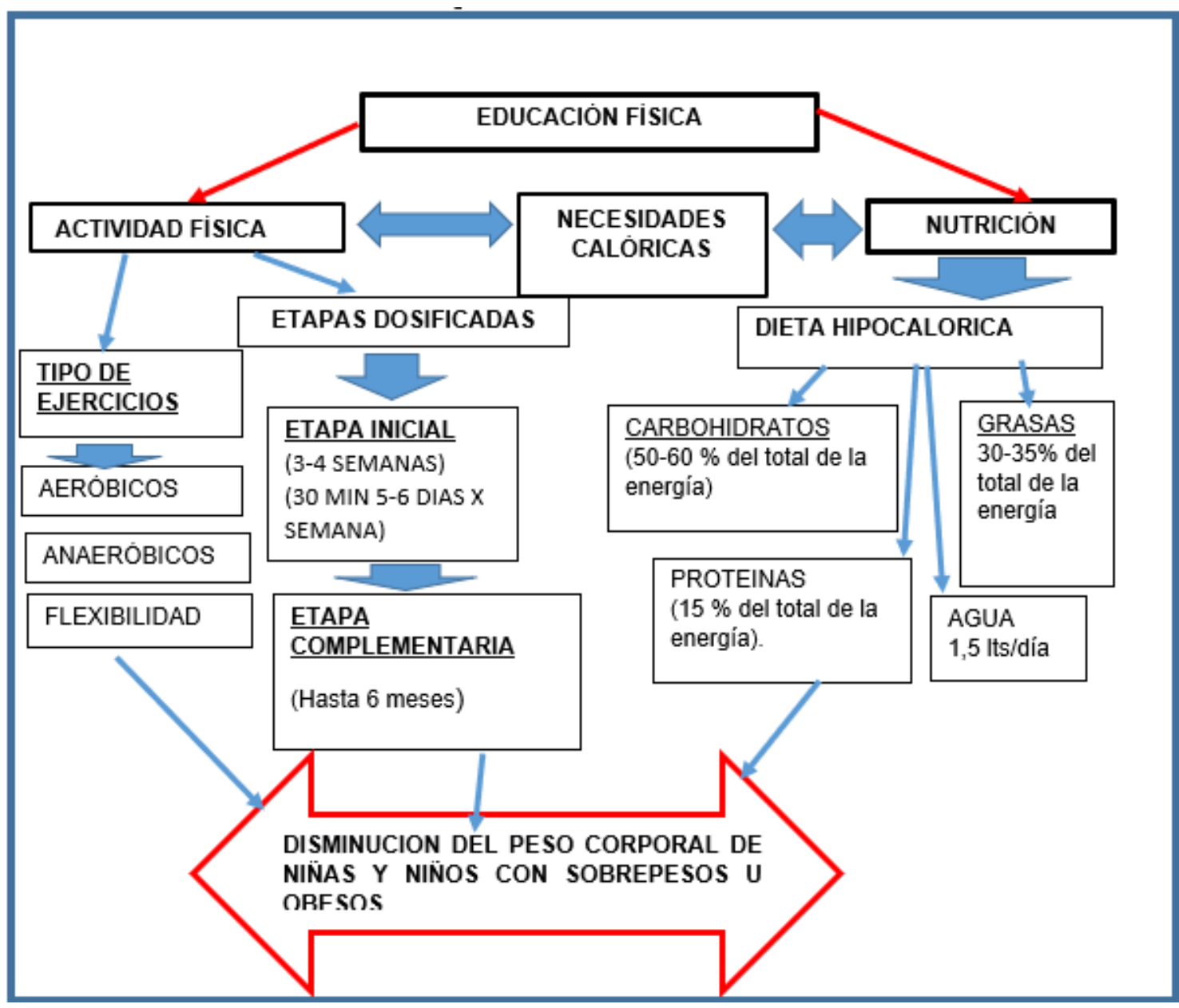

Fuente: Elaboración propia.

\section{Conclusiones:}

- A modo de conclusión podemos expresar que, resulta muy importante reflexionar en torno al tema tratado desde una visión multidisciplinar conducente al desarrollo de acciones preventivas que involucren el sujeto, el contexto socio familiar y educativo.

- Es imprescindible lograr una cultura educativa que permita comprender que la disminución del sobrepeso o la obesidad en los niños, niñas y adolescentes en las edades entre los 5 y 18 años, es un tema altamente necesario e importante y solo puede lograrse a través de la aplicación de un programa estructurado de manera multidisciplinar, que vincule la actividad física planificada basada en una praxis pedagógica organizada y coherente con los alcances y propósitos a lograr y que además, incluya una nutrición adecuada que respete las necesidades calóricas de 
forma tal que no afecte la salud de los niños y niñas así como un control periódico de los tipos de ejercicios, carga e intensidad de los mismos y la posibilidad de su continua adaptación en función de los resultados esperados. Los autores quieren agradecer a la Universidad Técnica de Ambato, a la Unidad Operativa de Investigación y Desarrollo (FCHE) y al grupo de Investigación Promoción de la Calidad de Vida por el apoyo en la ejecución de este trabajo investigativo.

\section{Referencias bibliográficas:}

Aguilar Cordero, A. Ortegón Piñero, N. Mur Villar, J. C. Sánchez García, J. J. García Verazaluce, I. García García5y A. M. Sánchez López. (2014). Programas de actividad física para reducir sobrepeso y obesidad en niños y adolescentes; revisión sistemática. Revista Nutrición Hospitalaria, vol 30 Nro 4. Madrid oct.2014. versión On-line ISSN 1699-5198versión impresa ISSN 0212-1611

Barbany JR. Adaptaciones Fisiológicas al entrenamiento. En: Barbany JR (Ed). Fisiología del Ejercicio Físico y del Entrenamiento. Editorial Paidotribo. Barcelona. 2002. Pág 155-167.

Barbany, M., Foz. M. "Obesity: Concept, clasiffication and diagnosis"; SEEDO, ANALES Sin San Navarra, 2002, Vol. 25, Suplemento I, page 7- 16.

Butterfield GE y Calloway DH. (1984) "Physical activity improves protein utilization". Brt J Nutr; 51: 171-84.

B. Sobradillo, A. Aguirre, U. Aresti, A. Bilbao, C. Fernández Ramos, A. Lizárraga, et al. Curvas y tablas de crecimiento (estudios longitudinal y transversal). Fundación Faustino Orbegozo Eizaguirre, Bilbao (2004).

Cevallos Paredes K; Castillo Ruíz L; Educación física: actividad física y nutrición. Consideraciones para mejorar la praxis pedagógica. OLIMPIA. Revista de la Facultad de Cultura Física de la Universidad de Granma.Vol.15 No.50, julio septiembre 2018. ISSN: 1817-9088. RNPS: 2067.

Encuesta Nacional de Nutrición y Salud (ENSANUT 2011-2013)- Instituto Nacional de Estadística y Censos de Ecuador. 2013.

Foz, M. Formiguera, X. Obesidad. España: Harcourt Brace. 1998. 25-109.

Fox, E. Sports Physiology. Second Edition. USA: CBS College Publishing. 1984. 40-57.

Janiszewski, P. M., \& Ross, R. (2009).The utility of physical activity in the management of global cardiometabolic risk. Obesity, 17, 3-S14.doi: http://dx.doi.org/10.1038/oby.2009.382 
Manson, J. Greenland, P. LaCroix, A. Stefanik, M. Mouton, C. Oberman, A. et al. Walking Compared with Vigorous Exercise for the Prevention of Cardiovascular Events in Women. N Engl J Med. 2002 Sep 5; 347 (10): 716- 725

Micheli, L. Team Physician Manual. Hong Kong: Lippincott Williams and Wilkins Asia. 2001. 49-75. www.emedicine.com/med/topic1653.htm. Obesity. January 21 2002. Medscape is the leading online global destination for physicians and healthcare professionals worldwide.

M.DeOnis, A.W. Onyango, E. Borghi, A. Siyam, C. Nishida, J. SiekmannDevelopment of a WHO growth reference for school-aged children and adolescents. Bull WHO, 85 (2007), pp. 660-667. Bulletin of the World Health Organization 2007; 85:660-667. Departamento de Nutrición, Organización Mundial de la Salud, 20 Avenue Appia, 1211 Ginebra 27, Suiza.

Nelson, ME, Rejeski, WJ, Blair SN, Duncan, PW, Judge JO, King AC, Castaneda-Sceppa C. Physical activity and public health in order adults: Recommendation from the American College of Sports Medicine and the American Heart Association. Circulation, 2007, 116(9): 1094-1105.

Organización Mundial de la Salud, "10 datos sobre la obesidad”, Febrero de 2010, OMS, $\begin{array}{lllllll}\text { consultado el } & 30 & \text { de } & \text { junio }\end{array}$ http://www.who.int/features/factfiles/obesity/facts/es /index1.html.

Ortega Sánchez-Pinilla Ricardo. Medicina del Ejercicio Físico y el Deporte para la Atención a la Salud. Ed. Díaz de santos. Madrid. 1992. Disponible en: http://books.google.com.co/books?id=VHLaCDRxx

QAC\&pg=PR19\&dq=\%22ORTEGA ++ S\%C3\%81NC

HEZ-

PINILLA\%22\&lr=\&as_brr=0\&ei=I8pmSjhGJewyATA7DbDw\&rview=1\&cd=5 $\# \mathrm{v}=$ onepage $\& \mathrm{q}=\& \mathrm{f}=\mathrm{false}$.

Pollock, Michael L. Willmore, Jack H. Exercise in health and disease: Evaluation and prescription for prevention and rehabilitation. Second editions. W.B. Saunders Philadelphia. 1990.

Roldán Aguilar E, Muñoz Contreras A, Ochoa Alzate D. Relationship between Physical Activity and Body Mass Composition in People with Cardiovascular Risk. In: Sedentary Behavior: Physiology, Health Risks and Interventions. Ed. Nova Publisher. New York. 2010.

Roldán Aguilar Elkin, Rendón S. D; Revista Politécnica ISSN 1900-2351, año 9, Número 16, Páginas 75-84, 2013. 
Roldán A, Elkin. Tres años en Busca del conocimiento. Documentos de apoyo al área biomédica. SIAFYS (semillero de investigación de actividad física y salud, Ed. Politécnico Colombiano JIC. ISBN: 978-958-9090-09-1. 2007. Páginas 217

S.E. Barlow, and the Expert Committee Pediatrics Expert Committee recommendations regarding the prevention, assessment, and treatment of child and adolescent overweight and obesity: Summary report

Pediatrics, 120 (2007), pp. 164-192

Wilmore, J. H. J., Després, ,J.P.J.P., Stanforth, P. R. P., Mandel, S. S., Rice, T. T., Gagnon, J. J., Bouchard, C. C. (1999). Alterations in body weight and composition consequent to $20 \mathrm{wk}$ of endurance training: The HERITAGE family study. The American Journal of Clinical Nutrition, 70(3), 346- 352. Retrieved from http://search.proquest.com/docview/70022718?acc ountid=43746.

Wong M, Murillo G. Fundamentos fisiopatológicos de la obesidad y su relación con el ejercicio. Revista Acta médica costarricense vol-46 supl. 1. San José. Oct.2004.

\section{Liencia}




\section{PARA CITAR EL ARTÍCULO INDEXADO.}

Sailema Torres, Ángel A., Sailema Rios, J. P., Sailema Ríos, Ángelo D., \& Sailema Torres, M. (2020). Nutrición, sobrepeso y obesidad en la edad infantil: Su relación con la actividad física deportiva. Explorador

Digital, 4(3),

62-77. https://doi.org/10.33262/exploradordigital.v4i3.1280

\section{\Ciencia}

El artículo que se publica es de exclusiva responsabilidad de los autores y no necesariamente reflejan el pensamiento de la Revista Explorador Digital.

El artículo queda en propiedad de la revista y, por tanto, su publicación parcial y/o total en otro medio tiene que ser autorizado por el director de la Revista Explorador Digital.
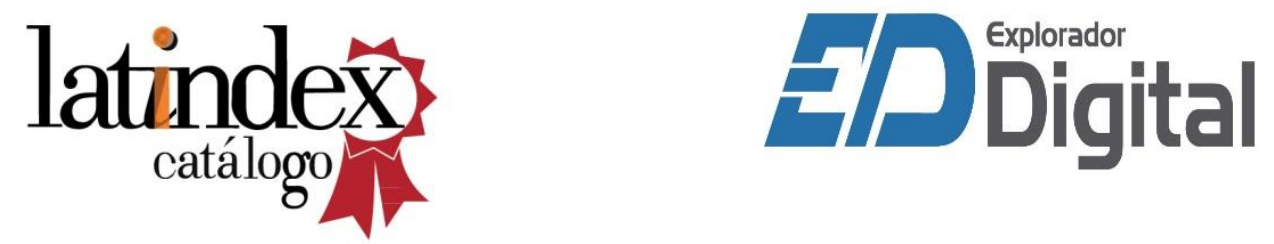